\title{
Metagenomics reveals unexplored Methanobrevibacter diversity in ancient dental calculus
}

Lena Granehäll

Kun D. Huang

Adrian Tett

Paolo Manghi

Alice Paladin

Niall O'Sullivan

Omar Rota-Stabelli

Nicola Segata

Albert Zink

Frank Maixner

\section{Video Byte}

Keywords: ancient DNA, ancient dental calculus, oral microbiome, metagenomics, de novo assembly, Methanobrevibacter, tooth, dental plaque, human remains, Neolithic, Early Middle Ages, Microbiome, archaea, taxonomy, microbial diversity, microbial evolution, anthropology, Trentino-South Tyrol, microbe, phylogenetics

Posted Date: October 13th, 2021

DOI: https://doi.org/10.21203/rs.3.rs-967535/v1

License: (c) (i) This work is licensed under a Creative Commons Attribution 4.0 International License. Read Full License 


\section{Abstract}

Dental calculus, or mineralized dental plaque, preserves various microfossils and biomolecules, including DNA. Dental calculus from ancient human remains therefore contains information about the oral microbiomes, health, and diets of our ancestors. However, little is known about the non-bacterial microbes in ancient calculus. In a new study, researchers used metagenomics to study calculus from 20 sets of human remains dating to the Neolithic period through the Early Middle Ages. Compared with modern calculus in publicly available datasets, the ancient calculus had a much higher abundance of archaea belonging to the phylum Euryarchaeota. Compared with modern calculus in publicly available datasets, the ancient calculus had a much higher abundance of archaea belonging to the phylum Euryarchaeota, specifically archaea in the genus Methanobrevibacter. The only known Methanobrevibacter species in modern calculus, M. oralis, was scarce in the ancient samples, but two new Methanobrevibacter species (TS-1 and TS-2) were detected. The three species were found across a broad geographic range and exhibited changes in diversity with time. Although studies on more samples, particularly modern samples, are needed, the results demonstrate the value of metagenomics for research on microbial diversity and evolution in ancient samples and suggest that the Methanobrevibacter species in the human oral microbiome have shifted over time. 